\title{
Evaluación retrospectiva de la vigilancia de Streptococcus pneumoniae causante de enfermedades invasoras en adultos de la Región Metropolitana-Chile: 2000-2006
}

\author{
Aurora Maldonado B., Mabel Seoane M., Oriana San Martín B., Juan C. Hormazábal O. y Rosanna Lagos Z.
}

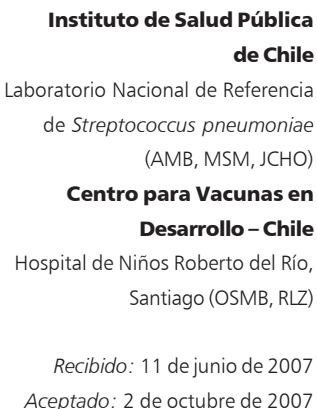

Correspondencia a: Rosanna Lagos Zuccone rlagos@adsl.tie.cl

\section{Retrospective assessment of laboratory surveillance for invasive Streptococcus pneumoniae isolates from adults of the Metropolitan Region (Chile): 2000-2006}

This study assessed the performance of the national norm for laboratory surveillance of invasive Streptococcus pneumoniae in adults of the Metropolitan Region (Chile). Material and Methods. We reviewed all notifications of isolates from patients $\geq 15$ years of age received by the Institute of Public Health of Chile during the period 2000-2006, submitted by health care facilities of the study region. We also reviewed the original records of sterile fluid cultures, in 14 public adult hospitals. Results. We found documentation of 1429 invasive S. pneumoniae isolates recovered from adult patients, including 1095 reported and 334 notreported isolates. A 33\% under-reporting rate was estimated for the 14 hospitals where local laboratory records were inspected. Age and clinical diagnosis were omitted in $23 \%$ and $78 \%$ of the notifications, respectively. Among 303 isolates from patients $\geq 65$ years of age that were investigated with Quellung reaction, $235(78 \%)$ had capsular serotypes represented in the 23-valent polysacharide vaccine. Conclusions. The Ministry of Health of Chile announced implementation of 23-valent vaccine immunization program for the elderly. In the perspective of future evaluations of the impact of this intervention, the results of this study indicate the need of reinforcing adherence and improving the quality of notifications of invasive S. pneumoniae.

Key words: Laboratory surveillance; invasive Streptococcus pneumoniae; adults.

Palabras claves: Vigilancia basada en laboratorios; Streptococcus pneumoniae invasor; adultos.

\section{Introducción}

$\mathrm{L}$ as enfermedades invasoras causadas por Streptococcus pneumoniae fueron incorporadas en la nómina de enfermedades objeto de vigilancia de laboratorio en Chile, a partir del 8 de noviembre de 1999, mediante el Decreto Supremo $N^{\circ} 712$. Para los efectos de esta vigilancia, el reglamento establece que todos los laboratorios asistenciales del país, públicos o privados, deben enviar las cepas de $S$. pneumoniae recuperadas de fluidos corporales, normalmente estériles, al Instituto de Salud Pública (ISP), acompañadas de una ficha epidemiológica $a d-h o c$, en la cual deben consignar datos de identificación del paciente, diagnóstico clínico, fecha de obtención y tipo de muestra que dio origen al aislado.

Sucesivos estudios epidemiológicos realizados en la Región Metropolitana (RM) han permitido colaborar con los establecimientos pediátricos en las tareas propias de la notificación y la referencia de las cepas al ISP, y reforzar el cumplimento de la norma ministerial de vigilancia de $S$. pneumoniae responsable de proce- sos invasores en niños bajo 15 años de edad ${ }^{1-5}$. La vigilancia de los aislados detectados en adultos, en cambio, se ha desarrollado sólo a través de los procedimientos rutinarios, con los recursos habitualmente disponibles en los laboratorios clínicos y en el ISP.

Hasta ahora, no ha habido un intento por analizar el funcionamiento de la vigilancia de $S$. pneumoniae causante de procesos invasores en adultos. Una evaluación de este tipo parece especialmente necesaria y oportuna en el momento actual, considerando que el Ministerio de Salud ha resuelto la implementación de un programa de inmunización con vacuna neumocóccica polisacárida 23-valente para la población de 65 años o más. Aunque la mayoría de las enfermedades causadas por este patógeno no llega a tener documentación bacteriológica, el seguimiento de los aislados de procesos invasores que son reportados al ISP será un elemento crítico -y tal vez el único disponible- para evaluar el impacto de dicho programa.

Este reporte resume observaciones sobre la calidad y la cobertura de la vigilancia de laboratorio para enfermedades neumocóccicas invasoras en sujetos de 15 
años o más, derivadas de una revisión de los datos disponibles en el Laboratorio Nacional de Referencia de S. pneumoniae del ISP y en los laboratorios de bacteriología de 14 hospitales estatales de la RM.

\section{Métodos}

Limites de la revisión y documentos fuente. El estudio abarcó el período de siete años, comprendido entre el 1 de enero de 2000 y el 31 de diciembre de 2006; se limitó a la RM y utilizó tres tipos de registros históricos: la base de datos del Laboratorio Nacional de Referencia de S. pneumoniae del ISP, los libros de laboratorios de bacteriología de 14 de los 18 hospitales estatales que atienden adultos en la RM y las fichas clínicas y/o archivos computacionales de egreso de los mismos hospitales. No se revisaron registros fuente en establecimientos privados o institucionales, en la Asistencia Pública, el Hospital del Tórax, el Instituto Traumatológico ni el Hospital de Buin. Los establecimientos asistenciales incluidos en la revisión, dan cuenta de $80 \%$ de las hospitalizaciones de adultos atendidas en establecimientos estatales de la RM durante el periodo del estudio.

Procedimiento de revisión. La base de datos del Laboratorio de Referencia fue filtrada para extraer los registros de cepas de $S$. pneumoniae recibidas entre el 1 de enero de 2000 y el 31 de diciembre de 2006, provenientes de los establecimientos ya señalados. A continuación, se eliminaron los registros repetidos (por ejemplo, réplicas de un misma cepa o aislados múltiples de un mismo paciente) y también aquellos correspondientes a muestras clínicas no estériles (nasofaríngeas, expectoración, secreción conjuntival, etc). Finalmente, se borraron los registros de cepas aisladas de pacientes bajo 15 años de edad. Esta selección dio origen a un archivo con 1.095 registros consistentes con los límites de tiempo, edad y procedencia definidos para el estudio.

Previa autorización de las jefaturas correspondientes, enfermeras del Centro para Vacunas en Desarrollo-Chile (CVD-Chile) visitaron los laboratorios de bacteriología de 14 hospitales estatales que atienden adultos en la RM, para revisar los registros de cultivos de fluidos estériles practicados durante el período de 2000 2006. A partir de esta revisión, confeccionaron una nómina de todos los aislados de $S$. pneumoniae detectados en procesos invasores y documentados en estos 14 laboratorios, incluyendo datos de identificación, diagnóstico, fecha de obtención y tipo de muestra clínica. Por último, en los mismos establecimientos intentaron completar datos de edad y diagnóstico clí- nico que no estaban consignados en la base de datos del ISP ni en los libros de los laboratorios de bacteriología, mediante revisión de fichas clínicas y/o registros computacionales de egresos hospitalarios.

Serotipos capsulares. Los datos de serotipos fueron obtenidos de los registros del Laboratorio de Referencia del ISP. Los serotipos capsulares fueron determinados mediante reacción de Quellung (hinchamiento capsular), con un kit Pneumotest ${ }^{\circledR}$ (Staten Serum Institute, Copenhagen) compuesto por 12 antisueros polivalentes y 44 factores; el kit permite identificar 45 serotipos específicos, incluyendo los 23 representados en la formulación de la vacuna neumocóccica polisacárida 23-valente. La técnica fue realizada por personal entrenado, bajo procedimientos de control de calidad externo de la red SIREVA ${ }^{6}$. La proporción anual de cepas referidas al ISP que fueron serotipificadas varió en función de la dotación de personal y de la disponibilidad de antisueros, pero no hubo criterios preestablecidos (por ejemplo, clínicos o microbiológicos) para seleccionar las cepas que fueron sometidas a este estudio.

Análisis. Este estudio descriptivo examinó el número anual de aislados de $S$. pneumoniae procedente de procesos invasores, enviados al ISP desde establecimientos asistenciales de la RM; la distribución etaria y el tipo de muestras que dio origen a los aislados. El nivel de adherencia a la norma de vigilancia fue evaluada en 14 (de 18) hospitales estatales de la RM, por contraste entre el total de aislados encontrados en los registros de los respectivos laboratorios asistenciales versus los encontrados en la base de datos del ISP.

La frecuencia de serotipos capsulares identificados en la colección de cepas serotipificadas por el Laboratorio Nacional de Referencia fue analizada con respecto a su representación en la vacuna neumocóccica polisacárida 23-valente. Por último, se estimó la incidencia promedio anual de enfermedad neumocóccica invasora en el período 2004-2006, en población de 15 a 44 años; 45 a 64 años y $\geq 65$ años de la RM.

\section{Resultados}

Total de casos detectados, edades y formas clínicas. Los registros del Laboratorio Nacional de Referencia arrojaron 1.095 notificaciones de $S$. pneumoniae implicados en procesos invasores correspondientes a pacientes de 15 años o más, incluyendo 792 enviadas desde establecimientos estatales y 303 provenientes de establecimientos privados (Tabla 1). En los 14 laboratorios de bacteriología visitados se encontraron re- 


\begin{tabular}{|c|c|c|c|c|c|}
\hline \multirow[t]{2}{*}{ Años } & \multicolumn{2}{|c|}{ Estatales } & \multicolumn{2}{|c|}{ Privados o Institucionales } & \multirow[b]{2}{*}{ Total } \\
\hline & $\mathbf{n}$ & $\%$ & $\mathbf{n}$ & $\%$ & \\
\hline 2000 & 97 & 84,3 & 18 & 15,7 & 115 \\
\hline 01 & 118 & 80,8 & 28 & 19,2 & 146 \\
\hline 02 & 89 & 67,9 & 42 & 32,1 & 131 \\
\hline 03 & 115 & 73,2 & 42 & 26,8 & 157 \\
\hline 04 & 128 & 74,0 & 45 & 26,0 & 173 \\
\hline 05 & 125 & 67,6 & 60 & 32,4 & 185 \\
\hline 06 & 120 & 63,8 & 68 & 36,2 & 188 \\
\hline Total & 792 & 72,3 & 303 & 27,7 & 1.095 \\
\hline
\end{tabular}

Tabla 2. Distribución de 1.429 cepas de Streptococcus pneumoniae invasor aisladas de pacientes de 15 años o más, según establecimiento y tipo de muestra clínica: Región Metropolitana, 2000-2006

\begin{tabular}{|c|c|c|c|c|}
\hline \multirow{3}{*}{$\begin{array}{l}\text { Tipo de muestra } \\
\text { clínica }\end{array}$} & \multicolumn{4}{|c|}{ Tipo de establecimientos } \\
\hline & \multicolumn{2}{|c|}{ Estatales } & \multicolumn{2}{|c|}{ Privados o institucionales } \\
\hline & $\mathbf{n}$ & $\%$ & $\mathbf{n}$ & $\%$ \\
\hline Sangre & 901 & 80,0 & 273 & 90,1 \\
\hline Líquido céfalo-raquídeo & 104 & 9,2 & 19 & 6,3 \\
\hline Líquido pleural & 60 & 5,3 & 7 & 2,3 \\
\hline Líquido peritoneal & 38 & 3,4 & 2 & 0,7 \\
\hline Líquido osteo-articular & 11 & 1,0 & 1 & 0,3 \\
\hline Otros fluidos estériles* & 12 & 1,1 & 1 & 0,3 \\
\hline Total & 1.126 & 100 & 303 & 100 \\
\hline
\end{tabular}

\begin{tabular}{|c|c|c|c|c|c|c|}
\hline Años & $\begin{array}{c}15 \text { a } 44 \\
\text { años }\end{array}$ & $\begin{array}{c}45 \text { a } 64 \\
\text { años }\end{array}$ & $\begin{array}{l}65 \text { años } \\
\text { o más }\end{array}$ & $n^{S}$ & $\%$ & Total \\
\hline 2000 & 33 & 41 & 40 & 30 & 20,8 & 144 \\
\hline 01 & 42 & 43 & 73 & 28 & 15,1 & 186 \\
\hline 02 & 33 & 48 & 57 & 24 & 14,8 & 162 \\
\hline 03 & 51 & 52 & 81 & 30 & 14,0 & 214 \\
\hline 04 & 60 & 54 & 82 & 47 & 19,3 & 243 \\
\hline 05 & 68 & 59 & 57 & 55 & 23,0 & 239 \\
\hline 06 & 47 & 68 & 98 & 28 & 11,6 & 241 \\
\hline Total & 334 & 365 & 488 & 242 & 16,9 & 1.429 \\
\hline
\end{tabular}

gistros de 334 casos adicionales. La Tabla 2 muestra la distribución de los 1.429 casos detectados durante el período 2000-2006 (notificados y pesquisados en los laboratorios clínicos), según el tipo establecimiento y muestra clínica que dio origen a cada aislado.

La mayoría de las cepas (78,3\%) fue referida al ISP sin diagnóstico clínico, mientras que el dato de edad fue omitido en $22,7 \%$ de las notificaciones. Los libros de trabajo de los 14 laboratorios asistenciales visitados permitieron reducir la omisión de edad a 16,9\%, pero mostraron carencias de información clínica consistentes con las detectadas en la base de datos del ISP. Los intentos por obtener antecedentes de edad y diagnóstico de las historias clínicas y/o de los archivos computacionales de egreso hospitalario tuvieron escaso rendimiento, porque los datos de identificación personal disponibles (nombre, RUT, número de ficha, etc) eran casi siempre insuficientes o incorrectos para rastrear estos registros.

En conjunto, los registros del Laboratorio Nacional Referencia y de los 14 establecimientos asistenciales visitados permitieron constatar la evidencia bacteriológica de 1.429 episodios de infección neumocóccica invasora diagnosticados en el RM durante el período del estudio y determinar la edad de 1.187 (83\%) de los pacientes afectados. La Tabla 3 detalla la distribución de los casos por año y grupos de edad.

Este estudio retrospectivo no permitió obtener datos confiables acerca de las formas de presentación o el resultado clínico de los casos detectados.

Estimación del porcentaje de referencia de cepas de S. pneumoniae recuperadas de enfermedades invasoras en 14 hospitales estatales. La Tabla 4 muestra el porcentaje anual de aislados de $S$. pneumoniae correspondientes a procesos invasores, que son referidos al ISP, sobre el total encontrado en los registros de bacteriología de los 14 laboratorios asistenciales visitados. Algunos de los libros de trabajo habían sido eliminados, o no fueron habidos, de manera que no fue posible revisar la totalidad de los cultivos de fluidos estériles practicados durante el período 20002006. Estas lagunas de información fuente se concentraron en cuatro laboratorios y en los primeros cuatro años del estudio. Como consecuencia de ellas, los datos presentados en la Tabla 4 adolecen de subrepresentación del número de casos diagnosticados al nivel local y de sobre-estimación de la referencia al ISP, especialmente, para los años 2000 a 2003. Obviando estos cuatro años, el nivel promedio de notificación de $S$. pneumoniae recuperados en procesos invasores diagnosticados en estos 14 laboratorios, en el trienio 2004-2006, alcanzó a 67,3\% (IC95\%; 63,2 - 71,2). 
Distribución de serotipos. Al cierre de este reporte (mayo de 2007), el Laboratorio de Referencia Nacional había determinado los serotipos de 827 de las 1.095 cepas invasoras correspondientes al período, región y edades del estudio. Como se aprecia en la Tabla 5, la proporción de cepas serotipificadas durante el período 2000-2004 fluctuó entre 34 y 65\%, mientras que en el último trienio fue consistentemente superior a $95 \%$. Este aumento en la proporción de cepas serotipificadas obedeció a una mayor dotación de profesionales entrenados en la técnica de Quellung, y también a mejor abastecimiento de antisueros en el Laboratorio de Referencia. Pese a estas diferencias en el número de aislados sometidos serotipificación, el rango de variación del porcentaje anual de cepas con serotipos capsulares representados en la vacuna polisacárida 23-valente fue considerablemente estrecho (máximo $80,2 \%$ el año 2003, mínimo $71,2 \%$, el año 2005).

La Figura 1 muestra la distribución porcentual de serotipos capsulares en las 827 cepas investigadas y en los subgrupos provenientes de pacientes 15 a 44 , 45 a 64 y 65 o más años de edad. El porcentaje de cepas con serotipos representados en la vacuna polisacárida 23 -valente alcanzó a $75,5 \%$ en la muestra total, y fluctuó entre 68,3 y 77,6\%, en los aislados recuperados de pacientes de 45 a 64 y mayores de 65 años, respectivamente. El orden de frecuencia de los serotipos predominantes también varió entre estratos de edad.

Estimación de incidencia por grupos etarios. Las estimaciones de incidencia de enfermedad neumocóccica invasora fueron calculadas con base a los casos notificados y detectados en los laboratorios locales durante el período 2004-2006. Las omisiones de edad fueron corregidas asumiendo que los casos sin dato se distribuyeron en los tres tramos de interés ( 15 a 44 años; 45 a 64 años y 65 años o más) en igual proporción que aquellos para los cuales se pudo obtener el antecedente de edad. La incidencia promedio anual de casos notificados alcanzó a 3,7 por $10^{5}$ sujetos de 15

\begin{tabular}{|c|c|c|c|}
\hline \multirow[t]{2}{*}{ Años } & \multirow{2}{*}{$\begin{array}{l}\text { Número de aislados detectados en } \\
\text { los } 14 \text { laboratorios asistenciales }\end{array}$} & \multicolumn{2}{|c|}{ Cepas referidas al ISP } \\
\hline & & $\mathbf{n}$ & $\%$ \\
\hline 2000 & 119 & 90 & $<75,6^{(\star *)}$ \\
\hline 01 & 141 & 101 & $<71,6^{(\star *)}$ \\
\hline 02 & 114 & 83 & $<72,8^{(\star *)}$ \\
\hline 03 & 169 & 112 & $<66,3^{(* *)}$ \\
\hline 04 & 195 & 125 & 64,1 \\
\hline 05 & 173 & 119 & 68,8 \\
\hline 06 & 173 & 120 & 69,4 \\
\hline Total & 1.084 & 750 & $67,3^{(* * *)}$ \\
\hline \multicolumn{4}{|c|}{$\begin{array}{l}\text { *La revisión no incluyó la Asistencia Pública, el Instituto Traumatológico, el Hospital de Buin } \\
\text { ni el Hospital del Tórax. Las } 42 \text { cepas referidas al ISP desde estos } 4 \text { establecimientos están } \\
\text { excluidas de la columna "Cepas referidas al ISP". **En algunos hospitales no se encontraron } \\
\text { registros de todos los cultivos de fluidos estériles, por lo tanto el \% de referencia está sobre- } \\
\text { estimado. }{ }^{* * *} \text { Calculado con base al datos del trienio } 2004-2006\end{array}$} \\
\hline
\end{tabular}

Tabla 5. Porcentaje anual de las cepas de Streptococcus pneumoniae invasor referidas al ISP, serotipificadas por el laboratorio de referencia

\begin{tabular}{|rrrr|}
\hline Años & Número de cepas recibidas por el ISP & \multicolumn{2}{c|}{$\begin{array}{c}\text { Cepas } \\
\mathbf{n}\end{array}$} \\
\hline 2000 & 115 & 39 & 33,9 \\
\hline 01 & 146 & 96 & 65,8 \\
\hline 02 & 131 & 55 & 42,0 \\
\hline 03 & 157 & 101 & 64,3 \\
\hline 04 & 173 & 171 & 98,8 \\
\hline 05 & 185 & 184 & 99,5 \\
\hline 06 & 188 & 181 & 96,3 \\
\hline Total & 1.095 & 827 & 75,5 \\
\hline ISP: Instituto de Salud Pública de Chile & & \\
\hline
\end{tabular}

Tabla 6. Estimación de la incidencia anual de infección neumocóccica invasora en adultos de la Región Metropolitana: 2004-2006

\begin{tabular}{|c|c|c|c|c|c|c|c|}
\hline \multirow[b]{3}{*}{ Edades $^{(*)}$} & \multicolumn{7}{|c|}{ Promedio anual, 2004-2006 } \\
\hline & & \multicolumn{2}{|c|}{ Notificado } & \multicolumn{2}{|c|}{ Detectado ${ }^{(* * *)}$} & \multicolumn{2}{|c|}{ Corregido ${ }^{(* * * *)}$} \\
\hline & Población ${ }^{(* *)}$ & Casos & Incidencia $\times 10^{5}$ & Casos & Incidencia $\times 10^{5}$ & Casos & Incidencia $\times 10^{5}$ \\
\hline 15 a 44 años & 3.138 .822 & 53,0 & 1,7 & 71,1 & 2,3 & 77,0 & 2,5 \\
\hline 45 a 64 años & 1.305 .111 & 53,4 & 4,1 & 73,6 & 5,6 & 79,7 & 6,1 \\
\hline 65 años o más & 504.646 & 75,2 & 14,9 & 96,3 & 19,1 & 104,3 & 20,7 \\
\hline 15 años o más & 4.948 .579 & 181,6 & 3,7 & 241,0 & 4,9 & 261,0 & 5,3 \\
\hline
\end{tabular}


Figura 1. Principales serotipos de Streptococcus pneumoniae invasor identificados en pacientes de 15 años o más. Región Metropolitana, 2000-2006.
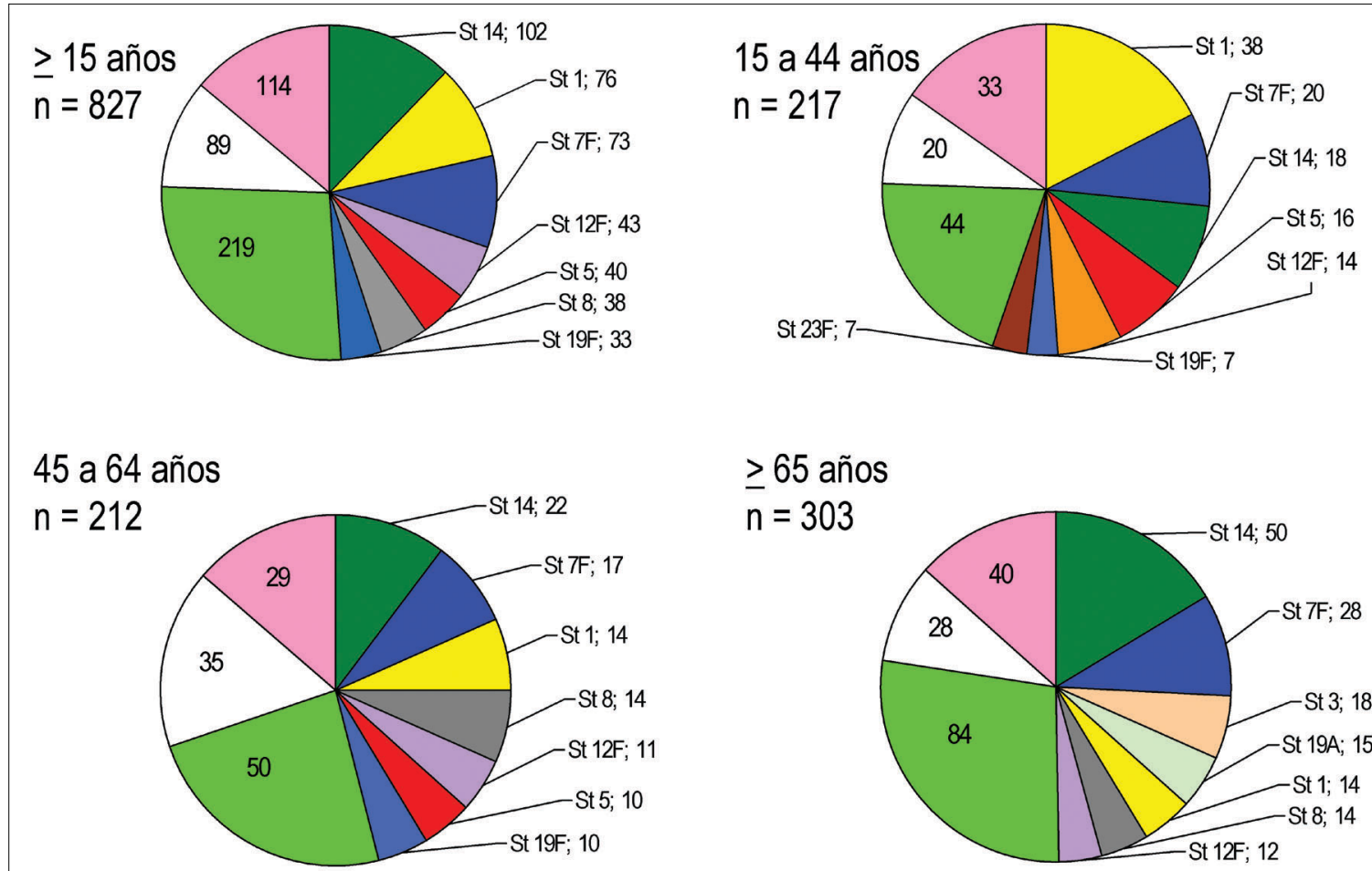

Otros serotipos contenidos en la vacuna 23-valente Serotipos/grupos no representados en la vacuna 23 -valente No tipificables con los antisueros disponibles

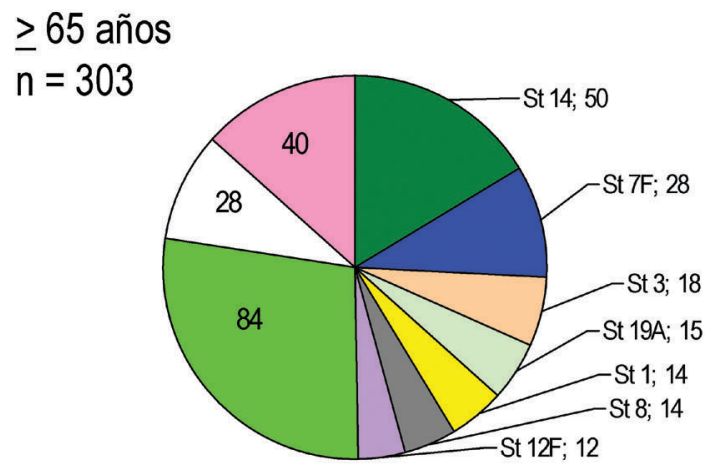

* Los números interiores representan el total de cepas en la categoría

* 95 cepas sin edad están representadas sólo en la figura superior-izquierda años o más. La estimación aumentó a 4,9 por $10^{5}$, al incorporar los 177 casos adicionales detectados en los registros de los 14 laboratorios visitados (Tabla 6, izquierda y centro, respectivamente). La sección derecha de la Tabla 6 muestra una estimación de incidencia corregida, aplicando a los casos notificados por los establecimientos cuyos registros de laboratorio no fueron revisados el factor de sub-referencia (33\%) encontrado en los 14 laboratorios donde sí se inspeccionaron los libros de bacteriología.

\section{Discusión}

Comparados con otros modelos de vigilancia, los sistemas basados en referencia de laboratorio poseen ventajas de practicabilidad y costos, pero también debilidades bien reconocidas, como posibles sesgos de derivación desde los laboratorios clínicos hacia los centros de referencia; variaciones temporales y geográficas en el cumplimento de la norma y, muy frecuentemente, omisión de datos demográficos indispensa- bles para permitir inferencias de tipo epidemiológico ${ }^{7-9}$. Esta evaluación de la vigilancia de $S$. pneumoniae causante de enfermedades invasoras en adultos de la RM refleja claramente estas limitaciones pero, a la vez, ilustra que la mayoría de ellas puede ser subsanada sin necesidad de aumentar la complejidad del sistema de vigilancia, simplemente, mejorando la calidad de los registros propios de la rutina asistencial, y la comunicación entre los actores que participan en la sospecha clínica, la documentación bacteriológica y la notificación de los casos.

Para evaluar la adherencia al reglamento de notificación y referencia de las cepas de $S$. pneumonaie recuperados en procesos invasores, revisamos los registros de cultivos estériles practicados en 14 hospitales estatales y contrastamos los aislados allí documentados con los encontrados en la base de datos del ISP. La revisión demostró sub-notificación de, al menos, $33 \%$ de los casos documentados durante el trienio 2004-2006, con variaciones considerables -de $12 \%$ hasta $59 \%$ - de un laboratorio a otro. Así, corresponde preguntarse si la información que puede generar el Labo- 
ratorio Nacional de Referencia (serotipos, susceptibilidad a antimicrobianos, etc) representa al universo de casos de enfermedad neumocóccica invasora que ocurrieron en adultos de la RM durante el período del estudio, o si en el envío de cepas existió algún sesgo que pudiera afectar la representatividad de estos datos.

Esta evaluación retrospectiva también reveló omisión de información clínica y datos de identificación incompletos o erróneos, en la mayoría de las notificaciones acumuladas en el ISP. Puesto que la cantidad y calidad de los datos disponibles en las notificaciones fueron consistentes con los encontrados en los registros originales de los respectivos aislados, al nivel de los laboratorios clínicos, concluimos que los errores y omisiones comenzaron antes del ingreso de las muestras sospechosas a los laboratorios asistenciales, muy probablemente, en las ordenes de investigación bacteriológica emitidas por los médicos. Este estudio demostró, en forma elocuente, las implicancias que pueden tener las falencias en procedimientos básicos de registro, sobre la calidad de la información que genera un sistema de vigilancia basado en referencia de laboratorio pasiva. En efecto, pudimos constatar la evidencia bacteriológica de 1.429 casos de enfermedad neumocóccica invasora; sin embargo, fue imposible obtener datos confiables acerca de las formas de presentación, las causas subyacentes, y el resultado clínico de estas enfermedades.

Aunque este estudio no permite informar sobre la adherencia a la norma de vigilancia de $S$. pneumoniae causante de enfermedades invasoras en establecimientos asistenciales privados o institucionales de la RM, llama la atención que el número anual de aislados notificados por establecimientos privados aumentó en forma sostenida a través de todo el período abarcado por la revisión, mientras que los reportados por los hospitales estatales se mantuvieron estables. Las causas que podrían explicar esta asimetría son numerosas, incluyendo participación creciente del sector privado en las prestaciones de salud. Sin embargo, antes de apelar a otras explicaciones, es necesario descartar que los establecimientos privados estén superando a los estatales, en lo que respecta al cumplimiento del reglamento de referencia de las cepas de S.pneumoniae aisladas desde procesos invasores.

La distribución de serotipos presentada en este reporte también podría ser cuestionada, toda vez que la serotipificación fue practicada en menos de $60 \%$ de los aislados detectados en la revisión (827 de 1.429). A favor de la representatividad de los datos, el porcentaje de cepas con serotipos representados en la vacuna 23 -valente $(624 / 827$, es decir $75,4 \%)$ resultó muy semejante al descrito entre los aislados recuperados de pacientes de 15 o más años de edad, en un estudio realizado en Temuco $(234 / 320,73,1 \%)^{10}$. Además, el rango de variación del porcentaje anual de cepas con serotipos representados en esta vacuna fue considerablemente estrecho (máximo 80,2\% el año 2003, mínimo 71,2\%, el año 2005).

No obstante las limitaciones discutidas arriba, este estudio es el primero que permite inferir estimaciones de incidencia con base poblacional, de infecciones neumocóccicas invasoras en adultos de la RM. Las estimaciones son conservadoras, porque las correcciones aplicadas al número de casos constatados fueron marginales, comparadas con las posibilidades de sub-detección inherentes a esta revisión de registros históricos. Basados en un seguimiento prospectivo de los casos diagnosticados en el Hospital Regional de Temuco, entre los años 1994 y 2004, Inostroza y cols reportaron una incidencia de $60 \times 10^{5}$ en adultos de 65 años o más, tres veces más alta que la estimada en este estudio retrospectivo ${ }^{10}$. Estas diferencias podrían derivar de las limitaciones metodológicas mencionadas; de una mayor sensibilidad en la pesquisa clínica y/o la investigación bacteriológica de los casos sospechosos en el hospital de Temuco, y también de diferencias reales del riesgo de enfermedad neumocóccica invasora entre ambas comunidades. Sin embargo, dado que el estudio de Temuco contabilizó todos los casos diagnosticados en el hospital regional, pero utilizó como denominador sólo la población de la comuna de ocurrencia $^{11}$, es muy posible que las cifras de incidencia estén inclinadas en el sentido opuesto a las de nuestro estudio, es decir, hacia la sobre-estimación. De hecho, las estadísticas del Servicio de Salud Araucanía Sur informan que aproximadamente la mitad de los egresos generados por el hospital regional corresponde a residentes de otras comunas de la Región ${ }^{11}$.

Con miras a la próxima implementación de un programa con vacuna neumocóccica polisacárida 23valente en adultos mayores, esta revisión señala la necesidad de corregir falencias y reforzar la adherencia a la norma nacional de vigilancia de $S$. pneumoniae causante de enfermedades invasoras y de incorporar procedimientos simples (como esta revisión) que permitan monitorear su cumplimiento a través del tiempo y a lo largo de todo el país. El impacto de esta medida deberá ser evaluado teniendo en cuenta las tasas de sub-notificación previas y posteriores a su implementación.

\section{Resumen}

Este estudio evaluó el funcionamiento de la norma nacional de vigilancia de Streptococcus pneumoniae recuperado desde procesos invasores en adultos de la 
Región Metropolitana-Chile. Material y Métodos: Se revisaron las notificaciones correspondientes a pacientes de 15 años o más, recibidas por el ISP en el período 2000-2006, emitidas por establecimientos asistenciales de la región. Paralelamente, se revisaron los resultados de cultivos de fluidos estériles practicados en 14 hospitales estatales que atienden adultos. Resultados: Se encontró documentación de 1.429 aislados de $S$. pneumoniae provenientes de procesos invasores en pacientes de 15 años o más, incluyendo 1.095 notificados y 334 no notificados. La tasa de subnotificación estimada para los 14 establecimientos visitados alcanzó a 33\%. La edad y el diagnóstico fueron omitidos en 23 y 78\% de las notificaciones, respectivamente. En 235 de 303 (78\%) cepas aisladas de pacientes de 65 años o más, investigadas mediante reacción de Quellung, se identificaron serotipos capsulares representados en la vacuna polisácarida 23-valente. Conclusiones: El Ministerio de Salud anunció un programa de inmunización con vacuna neumocóccica 23valente dirigido a los adultos mayores. Con miras a la futura evaluación del impacto de esta medida, los resultados de esta revisión señalan la necesidad de reforzar el cumplimento y la calidad de las notificaciones de $S$. pneumoniae causante de enfermedades invasoras.

\section{Referencias}

1.- Levine M, Lagos R, Levine O, Heitmann I, Enriquez N, Pinto M E, et al. Epidemiology of invasive pneumococcal infections in infants and young children in Metropolitan Santiago, Chile, a newly industrializing country. Pediatr Infect Dis J 1998; 17: 287-93.

2.- Lagos R, San Martín O, Erazo A, Avendaño A, Levine $\mathrm{M}$ y el Grupo de trabajo colaborativo para el diagnóstico de infecciones neumocóccicas del niño. Epidemiología de las enfermedades invasoras causadas por Streptococcus pneumoniae en niños chilenos: Proyecciones clínicas y de salud pública. Rev Chil Infect (2001); 18 (Supl 1): 15-21.

3.- Lagos R, Munoz A, Valenzuela M T, Heitmann I, Levine M M. Population-based surveillance for hospitalized and ambulatory pediatric invasive pneumococcal disease in Santiago, Chile. Pediatr Infect Dis J 2002; 21: 1115-23.

4.- Lagos R, Muñoz A, San Martín O, Heitmann I, Loyola H, Levine M M.
Secular variations in age incidence (Inc) and serotypes (St) causing invasive pneumococcal disease (IPD) in children 0 to 59 months of age (MoA) in the Metropolitan Region (MR), Chile. Abstract \# PO3.34; $5^{\text {th }}$ International Symposium on Pneumococci and Pneumococcal Diseases; Alice Springs, Australia, April 2006.

5.- Lagos R, Muñoz A, Hernández M, San Martín O, Seoane M, Maldonado A, et al. Relative invasive disease (ID) potential of Streptococcus pneumoniae (S.pn) of specific serotypes and serogroups, in Chilean infants and children from 0 to 24 months of age (MoA). Abstract \# PO3.63; $5^{\text {th }}$ International Symposium on Pneumococci and Pneumococcal Diseases; Alice Springs, Australia, April 2006.

6.- Di Fabio J L, Castañeda E, Agudelo C I, De la Hoz F, Hortal M, Camou T, et al. Evolution of Streptococcus pneumoniae serotypes and penicillin susceptibility in Latin America, Sireva-Vigía Group, 1993-1999. Pediatr Infect Dis J 2001; 959-67.
7.- Standaert S M, Lefkowitz L B, Horan J M, Hutcheson R H, Schaffner W. The reporting of communicable diseases: a controlled study of Neisseria meningitidis and Haemophilus influenzae type $\mathrm{b}$ infections. Clin Infec Dis 1995; 20: 30-6.

8.- Vergison A, Tuerlinckx D, Verhaegen J, Malfroot A, Belgian Invasive Pneumococcal Disease Study Group. Epidemiologic features of invasive pneumococcal disease in Belgian children; passive surveillance is not enough. Pediatrics 2006; 118: e801-9.

9.- Sleeman K, Knox K, George R, Miller E, Waight $\mathrm{P}$, Griffiths D, et al. Invasive pneumococcal disease in England and Wales: vaccination implications. J Infect Dis 2001; 183: 239-46.

10.- Inostroza J, Illesca V, Reydet P, Vinet A M, Ossa G, Muñoz S, et al. Ten-year surveillance of pneumococcal infections in Temuco, Chile. Implications for vaccination strategies. Clin Vaccine Immunol 2007; doi:10.1128/CVI.00379-06.

11.- http://www.minsal.cl/ Bases de datos de Egresos Hospitalarios 2003-2005. Último acceso 23 de Octubre de 2007. 